\title{
Physician Use of Patient-centered Weblogs and Online Journals
}

\author{
Julie Hillan, Department of Language, Literature and Communication, Rensselaer Polytechnic Institute, Troy, New York \\ and Corporate Communications, Marshfield Cinic, Marshfield, Wisconsin
}

\author{
REPRINT REQUESTS: \\ Julie Hillan \\ Marshfield Oinic \\ Mailstop: RL4 \\ 1000 North Oak Avenue \\ Marshfield, Wl 54449 \\ Telephone: 715-389-4974 \\ Fax: 715-389-5799 \\ Email: hillan.julie@marshfieldclinic.org
}

Clinical Medicine \& Research

Volume 1, Number 4: 333-335

(C)2003 Cinical Medicine \& Research

http://www.mfldclin.edu/clinmedres

\begin{abstract}
Online weblogs and journals developed by people affected by illness are popular vehicles for personal expression and peer emotional support. These sites can also offer insight to healthcare providers about patient opinions, habits and actions. These same media can also be effective and efficient tools for exchanging information between patients, providers, or between a patient and his/her provider(s).
\end{abstract}

\section{INTRODUCTION}

Social software, a relatively new term, is used to describe applications that allow groups of people to communicate and collaborate by means of a computer. Email, instant messaging and weblogs are examples of social software. This type of software has become a powerful creator of social capital by encouraging communication and interaction between users.

Weblogs, or "blogs," are public online web sites on which a person is able to post content regularly, with the newest entries being displayed first. Blogs are similar to personal home pages and newsletters except they often focus on a single topic. Blogs also use software that allows site visitors to post comments and feedback immediately to the site. Blogs are not only a conduit for the site creator's thoughts and feelings, but they can also provide a forum for ongoing discussion.

Originally, blogs were lists of web site addresses accumulated and posted by an individual. However, in recent years the term "blog" has also been used to describe frequently updated personal journals or diaries on the Internet. Some sites are hybrids, offering both lists of relevant Internet addresses and journal entries. In a nutshell, online journals focus on the person who created the site, and blogs commonly include lists of other sites he or she recommends. According to an estimate by Phil Wolff of blogcount.com, there are now more than 2 million blogs worldwide. ${ }^{1}$

Blogs and online journals are self-expressive media with sites devoted to a wide array of topics, ranging from news stories to healthcare. With the popularization of free and low-cost blogging tools that do not require special programming skills, many people are taking advantage of the blog and journal media as an easy-to-use form of online publishing. Most blog software offers password protection, thus the entire site can be blocked or specific sections can be hidden from public viewing. 
The personal perspective written in online journals and blogs offers physicians a unique channel for learning about the mental, emotional and physical state of people living with medical conditions and how these change over time. Comments by visitors who provide support, find a shared experience, or describe their triumphs and setbacks can be equally revealing. These ongoing forums are rich, anecdotal sources of individual experiences with disease progression, reaction to alternative and standard treatments, and opinions on healthcare and its effect on family members and lifestyle.

\section{THE POWER OF PATIENT BLOGS AND ONLINE J OURNALS}

Blogs and online journals have evolved as part of the consumer and patient empowerment trend that has characterized the Internet age. Common uses of these media created by people living with illness includes keeping a daily log of their disease state, progress and setbacks. Family and friends have an opportunity to "visit" regardless of geographical location, visitation restrictions, or even how well the author of the journal is feeling. Unless the site is password protected, anyone with an Internet connection can potentially access these public sites.

Blogs and online journals can create a sense of belonging to a larger social group for the user when they either create or read a site. They may interact by leaving comments or sending email messages. The sites allow users to connect on their own terms. Users may chose to participate actively (creating a site, posting comments, searching for sites of interest), or passively (reading sites only). Blogs and online journals overcome barriers such as isolation due to geography or physical limitations, as well as offering emotional outlets with varying degrees of anonymity.

\section{BLOGS AND ONLINE J OURNALS IN A HEALTHCARE SETTING}

Patient blogs and online journals are slowly being integrated into medical facilities, allowing patients to receive greetings from friends and family and to provide personal experience to people with the same medical condition. Using a password provided by the creator of the site, visitors can track the patient's condition and leave messages of support without interrupting care and privacy during emotionally and physically stressful times.

Physicians can review a patient's online diary and quickly address issues outside of regular appointments. Password protected blogs can focus on separate areas appropriate to the patient, his or her family, and/or care providers. In this way, medical providers can use blogs to exchange information with other members of a patient's care team. Facilities that lack an electronic medical records system or a readily available central chart system, can communicate efficiently through individual patient blogs. A blog can further connect providers through reports placed in a central location and through links to articles, categorized appropriately and allowing for contributions by everyone in the group or team.
Use of patient blogs and online journals by physicians and/or medical facilities has its drawbacks, with privacy issues being the most obvious. Furthermore, without the social interaction and non-verbal communication associated with human to human interaction, messages posted to a site are open to misinterpretation. Not every person is suited to this medium. While some patients may feel free to post their inner feelings, concerns and experiences through writing and publishing on the Internet, others will not find their personality or skills suited for this sort of public, or semipublic expression.

\section{A PATIENT-CENTERED J OURNAL/BLOG EXAMPLE}

Lisa Pierot's online journal, "The Metastatic Life," chronicles her experience as a cancer survivor. ${ }^{2}$ Pierot writes essays about her life from diagnosis through treatment and periods of recovery. Her revealing stories describe her fears and frustrations, feelings of isolation and loneliness, stages of disease acceptance, physical symptoms and personal struggles and achievements. Pierot clearly states her goals for the site's intended use on the Biography page.

My story is just that, mine; in sharing it, I hope I might bring understanding and hope into some of your lives. I pray that my writing about such a personal experience will encourage others to open up and share their feelings and experiences, too. With cancer, there are so many things to talk about, yet often we feel such isolation from the rest of the world.

An example of information from this site that a physician may find particularly insightful is Pierot's short essay titled "Dr. Harwin." Pierot discusses what kind of emotional support she felt necessary from a physician treating her. She details exactly how one provider fulfilled her needs when another did not. At one medical facility she was so disappointed in the care she received that she did not return. Pierot explains in her journal,

I told him that if there were one thing I could change about my experience at (the facility) it would be the doctors' way of relating to their patients...I told him what a difference it would have made if he'd bonded with me as a person during the whole ordeal."

Pierot also explains what she admires about her current physician: "We have a mutual respect and admiration that can only come from getting to know each other as people."

The type of information presented in these formats, one person's unique experience, will naturally be different from every other. The value for physicians in reading these individual expressions is to determine what resonates with their own experiences in working with their own patients. These unique forums-semi-anonymous, self-published, progressively updated-offer a range of anecdotal information for anyone who is interested in reading it. 


\section{CONCLUSION}

Computer-mediated communication is not able to replace the experience and nuance of human to human social networks; however, blogs and online journals can give voices and meaning to some people who would otherwise struggle to find an outlet. Social software is in common use, taking new forms, evolving and gaining popularity every day. As more people become computer literate and savvy, they will continue to find ways to connect with each other by means of the Internet.

\section{REFERENCES}

1. Wolff, P. Blogcount. Available at: http://dijest.com/bc/. Accessed August 26, 2003.

2. Peirot, L. The Metastatic Life. Available at: http://www.themetastaticlife.com/index.html. Accessed July 17, 2003. 